\title{
Análise do filme lacrimal e sua relação com a largiura da fenda palpebral e a exoftalmia na of talmopatia de Graves
}

\author{
Tear film analysis and its relation with palpebral fissure height and exophthalmos in \\ Graves'ophthalmopathy
}

\author{
Maria Vitoria Oliveira Moura Brasil ${ }^{1}$ \\ Oswaldo Ferreira Moura Brasil ${ }^{2}$ \\ Rodrigo Perez Vieira ${ }^{3}$ \\ Mário Vaisman ${ }^{4}$ \\ Octávio Moura Brasil do Amaral Filho ${ }^{5}$
}

Trabalho realizado no Hospital Universitário Clementino Fraga Filho, Universidade Federal do Rio de Janeiro (UFRJ).

${ }^{1}$ Residente do 30 ano do Serviço de Oftalmologia do Hospital Universitário Clementino Fraga Filho (UFRJ) Rio de Janeiro (RJ).

Residente do $3^{\circ}$ ano do Serviço de Oftalmologia do Hospital Universitário Clementino Fraga Filho (UFRJ). Rio de Janeiro (RJ).

${ }^{3}$ Pós-graduando nível Especialização do 30 ano do Serviço de Oftalmologia do Hospital Universitário Clementino Fraga Filho (UFRJ). Rio de Janeiro (RJ).

${ }^{4}$ Professor Adjunto do Departamento de Clínica Médica da Faculdade de Medicina, Chefe do Serviço de Endocrinologia do Hospital Universitário Clementino Fraga Filho (UFRJ). Rio de Janeiro (RJ).

${ }^{5}$ Professor Adjunto e Coordenador de Graduação do Departamento de Oftalmologia da Faculdade de Medicina, Chefe do Setor de Córnea e Doenças Externas do Hospital Universitário Clementino Fraga Filho (UFRJ). Rio de Janeiro (RJ).

Endereço para Correspondência: Maria Vitoria Oliveira Moura Brasil - Rua Maria Angélica, 270/403 - Rio de Janeiro (RJ) CEP 22461-150

E-mail:1lmv@unisys.com.br

Recebido para publicação em 13.09.2004

Versão revisada recebida em 02.03.2005

Aprovação em 23.05.2005

Nota Editorial: Depois de concluída a análise do artigo sob sigilo editorial e com a anuência dos Drs. Roberto Abucham e Mário Luiz Ribeiro Monteiro sobre a divulgação de seus nomes como revisores, agradecemos sua participação neste processo.

\section{RESUMO}

Objetivo: Avaliar qualidade do filme lacrimal pelo corante rosa bengala e sua estabilidade por meio do tempo de ruptura, relacionando com a largura da fenda palpebral e a exoftalmia em pacientes com oftalmopatia de Graves. Métodos: Foram estudados 54 olhos de 27 pacientes com oftalmopatia de Graves, tanto em fase inflamatória quanto em fase crônica. A avaliação consistiu de análise qualitativa do filme lacrimal pelo corante rosa bengala por meio da classificação de van Bijsterveld, análise da estabilidade do filme lacrimal pelo tempo de ruptura, medida da largura da fenda palpebral e exoftalmometria. A análise estatística foi realizada com o teste do Quiquadrado. Resultados: Entre os 27 pacientes estudados, 77,8\% eram do sexo feminino e 22,2\% do masculino. A idade média foi de 44,26 anos (DP 12,67). O tempo médio de doença foi de 5,85 anos (DP 4,47) e o de oftalmopatia foi de 5,81 anos (DP 5,37). Dos 54 olhos em estudo, 37\% apresentaram teste positivo pela escala de graduação de van Bijsterveld, $33,3 \%$ tempo de ruptura do filme lacrimal menor que 5 segundos, 57,4\% largura da fenda palpebral maior que $11 \mathrm{mme} 55,6 \%$ exoftalmometria maior que $19 \mathrm{~mm}$. Quando relacionamos o tempo de ruptura do filme lacrimal menor que 5 segundos com a largura da fenda palpebral maior que $11 \mathrm{~mm}$ encontramos odds ratio igual a $11,2(\mathrm{p}=0,0008)$. As demais relações estudadas não mostraram significância estatística. Conclusões: O olho seco diagnosticado pela coloração com rosa bengala e pelo tempo de ruptura do filme lacrimal ocorre com freqüência na oftalmopatia de Graves. A largura da fenda palpebral correlaciona-se com o tempo de ruptura do filme lacrimal na oftalmopatia de Graves. Seu aumento pode levar à instabilidade do filme lacrimal.

Descritores: Doença de Graves; Rosa bengala; Ceratite; Exoftalmia/diagnóstico; Lágrimas/fisiologia; Coloração e rotulagem

\section{INTRODUÇ̃̃̃O}

A oftalmopatia de Graves (OG) é a manifestação extra-tireiodiana mais comum da doença de Graves $(\mathrm{DG})^{(1)}$, representando a condição inflamatória mais freqüente dos tecidos orbitários e músculos extra-oculares ${ }^{(2)}$. Além disso, é a causa mais comum de exoftalmia, sendo responsável por, provavelmente, mais de $50 \%$ dos $\operatorname{casos}^{(3)}$.

Em 1995, foram propostos critérios diagnósticos para OG que incluíam: retração palpebral ou disfunção tireoideana objetiva associadas a exoftalmia, neuropatia óptica, ou acometimento dos músculos extra-oculares, desde que não atribuídos a outras causas ${ }^{(4)}$. 
As queixas relacionadas à doença da superfície ocular, assim como o diagnóstico de olho seco, são comuns na $\mathrm{OG}^{(5-6)}$.

Diversos testes diagnósticos foram desenvolvidos para avaliação de pacientes com ceratoconjuntivite seca, incluindo coloração com rosa bengala (RB), teste de Schirmer, tempo de ruptura do filme lacrimal (TRFL) e ensaio imunológico com lactoferrina $^{(7)}$.

A RB é um corante de cor vermelha forte que tem a propriedade de penetrar em células epiteliais mortas ou em sofrimento, corando-as. Sua grande indicação é demonstrar as alterações epiteliais que ocorrem na ceratoconjuntivite $\operatorname{seca}^{(8)}$.

Van Bijsterveld em 1969, criou uma escala de graduação para a coloração com RB que divide a superfície ocular em 3 zonas: conjuntiva bulbar nasal, córnea e conjuntiva bulbar temporal. Cada zona é avaliada pela densidade da coloração no valor de 0 a 3 , sendo 0 a ausência do corante e 3 sua impregnação confluente. Um valor total igual ou maior a 3 , para um olho, constituiria um teste positivo para olho $\operatorname{seco}^{(9)}$.

O objetivo deste trabalho foi estudar o papel da largura da fenda palpebral e da exoftalmia na avaliação da qualidade do filme lacrimal pelo corante RB e de sua estabilidade pelo TRFL.

\section{MÉTODOS}

Foram estudados 54 olhos de 27 pacientes com OG, em fases inflamatória ou crônica, em acompanhamento no Serviço de Endocrinologia, entre março e maio de 2004. O exame destes pacientes incluiu a medida da largura da fenda palpebral com régua milimetrada, exoftalmometria com exoftalmômetro de Hertel, coloração com RB e tempo de quebra do filme lacrimal com fluoresceína. A avaliação da coloração por RB foi feita através da classificação de van Bijsterveld, descrita anteriormente.

Foi também estudado, através de anamnese dirigida, a duração da DG, a duração da exoftalmia e sua presença como manifestação inicial da DG. A atividade da OG foi medida através do "Clinical Activity Score" (CAS).

Os resultados obtidos foram analisados utilizando teste do Qui-quadrado, com nível de significância estatística de 5\%.

\section{RESULTADOS}

Entre os 27 pacientes estudados, $21(77,8 \%)$ eram do sexo feminino e $6(22,2 \%)$ do masculino. A idade variou de 25 a 75 anos, com média de 44,26 (DP 12,67). Em relação à cor, 12 $(44,5 \%)$ eram brancos, $10(37 \%)$ pardos e $5(18,5 \%)$ negros.

O tempo de doença variou de 1 a 21 anos, com média de 5,85 anos (DP 4,47). O tempo de oftalmopatia também variou de 1 a 21 anos, com média de 5,81 anos (DP 5,37). Em 16 $(59,26 \%)$ pacientes o tempo de doença foi igual ao tempo de oftalmopatia, em $9(33,33 \%)$ o tempo foi maior e em $2(7,4 \%)$ foi menor. Desta forma, $18(66,66 \%)$ pacientes apresentaram a oftalmopatia como manifestação inicial da doença. Nos 2 pacientes em que a oftalmopatia foi notada antes da doença, houve retardo no diagnóstico da DG.
A avaliação da coloração pelo RB revelou pontuação variando de 0 a 6, com média de 2,07 (DP 1,63) nos 54 olhos em estudo, sendo que $20(37 \%)$ apresentaram teste positivo pela escala de graduação de van Bijsterveld.

O TRFL variou de 0 a 12 segundos, com média de 5,33 segundos (DP 2,71) nos 54 olhos em estudo, sendo que 18 $(33,3 \%)$ apresentaram TRFL menor que 5 segundos.

A largura da fenda palpebral variou de 7 a $19 \mathrm{~mm}$, com média de 12,53 mm (DP 2,64) nos 54 olhos estudados, sendo que $31(57,4 \%)$ apresentaram largura da fenda palpebral maior que $11 \mathrm{~mm}$.

A exoftalmometria variou de 12 a $28 \mathrm{~mm}$, com média de $19,94 \mathrm{~mm}$ (DP 3,85) nos 54 olhos estudados, sendo que 30 $(55,6 \%)$ apresentaram exoftalmometria maior que $19 \mathrm{~mm}$.

Várias relações foram testadas:

1. Teste positivo para RB com a largura da fenda palpebral maior que $11 \mathrm{~mm}$ onde o odds ratio $(\mathrm{OR})$ foi igual a 2,33 (0,63 a 9,14 IC 95\%, teste exato de Fisher, $\mathrm{p}=0,12$ ).

2. Teste positivo para RB com a exoftalmometria maior que $19 \mathrm{~mm}$ onde o OR foi igual a $0,70(0,20$ a 2,45 IC $95 \%$, teste exato de Fisher, $\mathrm{p}=0,36$ ).

3 . TRFL menor que 5 segundos com a largura da fenda palpebral maior que $11 \mathrm{~mm}$ onde o OR foi igual a 11,2 (2,04 a 109,91 IC $95 \%$, teste exato de Fisher, $\mathrm{p}=0,0008$ ) (Tabela 1 ).

4. TRFL menor que 5 segundos com a exoftalmometria maior que $19 \mathrm{~mm}$ onde o OR foi igual a $0,71(0,19$ a 2,59 IC $95 \%$, teste exato de Fisher, $\mathrm{p}=0,38$ ).

O CAS variou de 0 a 4, com média de 1,29 (DP 0,86).

\section{DISCUSSÃO}

Olho seco é a doença mais freqüente na prática oftalmológica em todo mundo. É um defeito qualitativo e/ou quantitativo do filme lacrimal. Entre suas diversas causas são incluídas as desordens das pálpebras que levam à distribuição inadequada da lágrima com formação de pontos secos e abrasões ${ }^{(10)}$.

Em 1983, foram avaliados 5 fatores considerados como potencialmente associados à exposição corneana: largura da fenda palpebral, exoftalmia, frequiência do piscar, lagoftalmo e acinesia óculo-palpebral ${ }^{(11)}$. Todos os pacientes eram portadores de DG e o método escolhido para avaliação das lesões da superfície ocular foi o corante RB. Os autores concluíram que a maior largura da fenda palpebral e o aumento da freqüência do piscar estariam associados a maior dano da superfície ocular.

\begin{tabular}{|c|c|c|c|}
\hline & TRFL $<5 s$ & TRFL $\geq 5 s$ & Total \\
\hline $\mathrm{FP}>11 \mathrm{~mm}$ & 16 & 15 & 31 \\
\hline $\mathrm{FP} \leq 11 \mathrm{~mm}$ & 2 & 21 & 23 \\
\hline Total & 18 & 36 & 54 \\
\hline \multicolumn{4}{|c|}{$\begin{array}{l}\text { FP }=\text { Fenda palpebral; TRFL }=\text { Tempo de ruptura do filme lacrimal. } \\
O R=11,2(2,04 \text { a } 109,91 \text { IC } 95 \% \text {, teste exato de Fisher, } p=0,0008)\end{array}$} \\
\hline
\end{tabular}


No nosso estudo não encontramos significância estatística quando relacionamos o RB com a largura da fenda palpebral e com a exoftalmia. No entanto, $37 \%$ apresentaram resultado positivo na escala de graduação de van Bijsterveld, constituindo teste positivo para olho seco. É importante salientar que $83,3 \%$ dos olhos tiveram ao menos 1 ponto (Tabela 2 ).

Desta forma, observamos uma alta freqüência de diagnósticos de olho seco pela coloração com RB na OG, ainda que não haja significância estatística quando relacionada à largura da fenda palpebral e à exoftalmia.

Em 1992, foi estudado o perfil do filme lacrimal em 30 pacientes com $\mathrm{OG}^{(12)}$. Os resultados obtidos mostraram que os valores do $\mathrm{pH}$ do filme lacrimal, coloração por fluoresceína, menisco lacrimal e teste de Schirmer foram comparáveis com os controles, indicando secreção lacrimal normal. O TRFL foi significativamente baixo na OG tardia, sugerindo instabilidade. A coloração com RB e com lissamina verde foi significativamente alta, indicando presença de ressecamento de células epiteliais, tanto na OG precoce como tardia.

Em nosso estudo, o TRFL menor que 5 segundos foi correlacionado com largura da fenda palpebral maior que $11 \mathrm{~mm}$. Esta relação foi estatisticamente significativa $(p=0,0008)$. Portanto, podemos considerar que o aumento da largura da fenda palpebral é fator de risco $(\mathrm{OR}=11,2)$ para a instabilidade do filme lacrimal na OG.

O mesmo não podemos dizer da relação entre o TRFL e a exoftalmia, pois a relação não foi estatisticamente significativa $(\mathrm{p}=0,38)$.

Quanto ao diagnóstico de olho seco pelo TRFL, 33,3\% dos pacientes apresentaram tempo inferior a 5 segundos, considerado diagnóstico. Ainda, 94,4\% apresentaram o tempo inferior a 10 segundos, considerado sugestivo do diagnóstico. Notamos então, uma frequiência importante de diagnósticos de olho seco pelo TRFL.

OCAS proposto em $1989^{(13)}$, avalia a atividade inflamatória da oftalmopatia e tem um alto valor preditivo para o resultado terapêutico cirúrgico ou com imunossupressores da doença ${ }^{(14-15)}$.

Consideramos o olho seco como uma doença que deve ser obrigatoriamente avaliada no portador da OG, uma vez que este pode ser confundido com a atividade da doença, até desnorteando o tratamento multidisciplinar que a patologia exige. Devemos ter em mente que a lubrificação adequada da

\begin{tabular}{|ccc|}
\hline Tabela 2. Freqüência de pontos na escala de graduação de van \\
Bijsterveld \\
Pontos & Freqüência & Porcentagem \\
0 & 9 & $16,7 \%$ \\
1 & 16 & $29,6 \%$ \\
2 & 9 & $16,7 \%$ \\
3 & 8 & $14,8 \%$ \\
4 & 8 & $14,8 \%$ \\
5 & 2 & $3,7 \%$ \\
6 & 2 & $3,7 \%$ \\
Total & 54 & $100,0 \%$ \\
\hline
\end{tabular}

superfície ocular tem grande importância nestes pacientes, não devendo ser esquecida quando avaliamos modalidades mais complexas de tratamento.

\section{ABSTRACT}

Purpose: To evaluate tear film quality by rose bengal staining and its stability by breakup time, relating with palpebral fissure height and exophthalmos in patients with Graves' ophthalmopathy. Methods: We studied 54 eyes of 27 patients with Graves' ophthalmopathy, either in the inflammatory or in the chronic phase of the disease. The evaluation consisted of tear film qualitative analysis by rose bengal staining using the van Bijsterveld grading scale, tear film stability analysis by breakup time, measurement of palpebral fissure height and exophthalmometry. Qui square test was used to perform statistical analysis. Results: Among 27 studied patients, $77.8 \%$ were females and $22.2 \%$ males. Mean age was 44.26 years (SD 12.67). Mean disease time was 5.85 years (SD 4.47) and mean ophthalmopathy time was 5.81 years (SD 5.37). Among 54 studied eyes, $37 \%$ had positive test by van Bijsterveld's grading scale, $33.3 \%$ tear film breakup time lower than 5 seconds, $57.4 \%$ palpebral fissure height greater than $11 \mathrm{~mm}$ and $55.6 \%$ exophthalmometry greater than $19 \mathrm{~mm}$. When relating tear film breakup time lower than 5 seconds with palpebral fissure height greater than $11 \mathrm{~mm}$ we found an odds ratio of 11.2 $(\mathrm{p}=0.0008)$. The remaining relationships did not show statistical significance. Conclusions: Dry eye diagnosed by rose bengal staining and tear film breakup time occurs frequently in Graves' ophthalmopathy. Palpebral fissure height correlates with tear film breakup time in Graves' ophthalmopathy. Its increase may lead to tear film instability.

Keywords: Graves' disease; Rose bengal; Keratitis/diagnosis; Exophthalmos; Tears/physiology; Staining and labeling

\section{REFERÊNCIAS}

1. Burch HB, Wartofsky L. Graves' ophthalmopathy: current concepts regarding pathogenesis and management. Endocr Rev. 1993;14(6):747-93.

2. Schworm HD, Heufelder AE, Kunze A, Welge E, Boergen KP. Clinical significance of saccade analysis in early active Graves' ophthalmopathy. Invest Ophthalmol Vis Sci. 2000;41(7):1710-8.

3. Rubin RM, Sadun AA. Ocular myopathies. In: Yanoff M, Duker JS. Ophthalmology. 2nd ed. Chicago, Il.: Mosby; 2004. p.1342-9.

4. Bartley GB, Gorman CA. Diagnostic criteria for Graves' ophthalmopathy. Am J Ophthalmol. 1995;119(6):792-5.

5. Cukierman E, Cavalcante LF, Henriques JLM, Yamane R. Orbitopatia tireóidea: análise clínica e epidemiológica. Rev Bras Oftalmol. 2000;59(8):578-85.

6. Ramos A, Maia M, Matsumoto LH, Matsumoto LT, Moreira Junior CA, Graf H. Alterações oftalmológicas na Doença de Graves: análise de 169 casos. Arq Bras Oftalmol. 1997;60(5):527-37.

7. Goren MB, Goren SB. Diagnostic tests in patients with symptoms of keratoconjunctivits sicca. Am J Ophthalmol. 1988;106(5):570-4.

8. Almada AT. Coloração vital. In: Belfort Jr R, Kara-José N. Córnea: clínica, cirúrgica. São Paulo: Roca; 1997. p.129-33.

9. Van Bijsterveld OP. Diagnostic tests in the Sicca syndrome. Arch Ophthalmol. 1969;82(1):10-4.

10. Freitas D, Sato EH, Souza LB. Olho seco. São Paulo: Phoenix; 2002. 
11. Gilbard JP, Farris RL. Ocular surface drying and tear film osmolarity in thyroid eye disease. Acta Ophthalmol (Copenh). 1983;61(1):108-16.

12. Khurana AK, Sunder S, Ahluwalia BK, Malhotra KC. Tear film profile in Graves' ophthalmopathy. Acta Ophthalmol (Copenh). 1992;70(3):346-9.

13. Mourits MP, Koornneef L, Wiersinga WM, Prummel MF, Berghout A, Van der Gaag R. Clinical criteria for the assessment of disease activity in Graves' ophthalmopathy: a novel approach. Br J Ophthalmol. 1989;73(8):639-44.
14. Mourits MP, Prummel MF, Wiersinga WM, Koornneef L. Clinical activity score as a guide in the management of patients with Graves' ophthalmopathy. Clin Endocrinol (Oxf). 1997;47(1):9-14.

15. Vaisman M, Violante AH, Conceição FL, Soares DV, Buescu A. High-dose intravenous pulse therapy with methylprednisolone and orbital irradiation in Graves' ophthalmopathy. Endocrinologist. 2001;11(1):53-61.

\section{6}

\section{CONGRESSO MUNDIAL DE OFTALM OLOGIA}

\section{Congresso Internacional de Oftalmologia XXVI Congresso Pan-Americano de Oftalmologia XVII Congresso Brasileiro de Prevenção da Cegueira}

\section{São Paulo - Brasil - 19-24 fevereiro 2006}

- Presença de todas as Sociedades Supranacionais, Internacionais e Nacionais

- 60 Sociedades de sub-especialidades do mundo inteiro representadas no programa científico

- 18 salas com 72 atividades por dia

- 630 apresentações científicas por dia

- Fórum Mundial de Jornais Científicos de O ftalmologia

- Fórum de O rganizações Não Governamentais

- 4 Subspecialty Days: Retina, Glaucoma, Catarata e Cirurgia Refrativa

\section{Inscrições de trabalhos on-line • Data limite: 28 de outubro de 2005 www.ophthalmology2006.com.br}

\begin{tabular}{|c|c|c|}
\hline & $\begin{array}{l}\text { A té } 15 \text { de novembro } \\
\text { de } 2005\end{array}$ & $\begin{array}{l}\text { No } \\
\text { local }\end{array}$ \\
\hline O ftalmologista sócio & $\mathrm{R} \$ 415,00$ & $\mathrm{R} \$ 550,00$ \\
\hline M édico & $\mathrm{R} \$ 715,00$ & $\mathrm{R} \$ 850,00$ \\
\hline $\begin{array}{l}\text { Residente e/ ou Aluno de Curso de Especialização } \\
\text { credenciado pelo CBO/Estudantes de Medicina }\end{array}$ & $\mathrm{R} \$ 210,00$ & $\mathrm{R} \$ 280,00$ \\
\hline O utros Profissionais, Técnico e A uxiliar & $\mathrm{R} \$ 240,00$ & $\mathrm{R} \$ 300,00$ \\
\hline Representante de ONGs & $R \$ 200,00$ & $R \$ 270,00$ \\
\hline A companhante & $\mathrm{R} \$ 150,00$ & $R \$ 200,00$ \\
\hline $\begin{array}{l}10^{\circ} \text { Congresso Internacional ISA-CLADE - São Paulo } \\
17 \text { a } 20 \text { de fevereiro de } 2005\end{array}$ & $\mathrm{R} \$ 150,00(*)$ & $\mathrm{R} \$ 150,00(*)$ \\
\hline
\end{tabular}

$(*)$ Valor a ser agregado à taxa do Mundial permitindo acesso às sessões científicas dos dois congressos. 